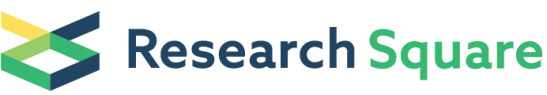 \\ Preprints are preliminary reports that have not undergone peer review. \\ They should not be considered conclusive, used to inform clinical practice, or referenced by the media as validated information.
}

\section{Symptomatic Features of 932 Hospitalized Patients with COVID-19 in Wuhan}

\section{Liang-Liang Sun}

Department of Endocrinology and Metabolism, Changzheng Hospital, Second Military Medical University, Shanghai, China. Optical Valley Branch of Maternal and Child Hospital of Hubei Province, Wuhan, China

\section{Jian Wang}

Department of Gastroenterology, Changzheng Hospital, Second Military Medical University, Shanghai, China

\section{Yu-Sheng Wang}

Department of Respiratory and Critical Care Medicine, Changzheng Hospital, Second Military Medical University, Shanghai, China

\section{Ping-Fang Hu}

Department of Gastroenterology, Changzheng Hospital, Second Military Medical University, Shanghai, China

\section{Zheng-Qing Zhao}

Optical Valley Branch of Maternal and Child Hospital of Hubei Province, Wuhan, China. Department of Neurology, Changzheng Hospital, Second Military Medical University, Shanghai, China

\section{Wei Chen}

Optical Valley Branch of Maternal and Child Hospital of Hubei Province, Wuhan, China. Department of Cardiovascular Medicine, Changzheng Hospital, Second Military Medical University, Shanghai, China

\section{Bei-Fang Ning}

Department of Gastroenterology, Changzheng Hospital, Second Military Medical University, Shanghai, China

\section{Chuan Yin}

Department of Gastroenterology, Changzheng Hospital, Second Military Medical University, Shanghai, China

\section{Yu-Shi Hao}

Department of Medical Psychology, Changzheng Hospital, Second Military Medical University, Shanghai, China

\section{Qiong Wang}

Department of Respiratory and Critical Care Medicine, Changzheng Hospital, Second Military Medical University, Shanghai, China

\section{Chen Wang}


Department of Respiratory and Critical Care Medicine, Changzheng Hospital, Second Military Medical University, Shanghai, China

\section{Ya-Long Liu}

Department of Respiratory and Critical Care Medicine, Changzheng Hospital, Second Military Medical University, Shanghai, China

\section{Cheng Chen}

Department of Respiratory and Critical Care Medicine, Changzheng Hospital, Second Military Medical University, Shanghai, China

\section{Ji-Zhong Yin}

Department of Respiratory and Critical Care Medicine, Changzheng Hospital, Second Military Medical University, Shanghai, China

\section{Hai Huang}

Optical Valley Branch of Maternal and Child Hospital of Hubei Province, Wuhan, China. Department of Respiratory and Critical Care Medicine, Changzheng Hospital, Second Military Medical University, Shanghai, China

\section{Wei-Fen Xie ( $\nabla$ weifenxie@medmail.com.cn )}

Changzheng Hospital: Shanghai Changzheng Hospital

\section{Research}

Keywords: COVID-19, SARS-CoV-2, Clinical characteristics, Symptomatic features

Posted Date: January 20th, 2021

DOl: https://doi.org/10.21203/rs.3.rs-148616/v1

License: (9) (1) This work is licensed under a Creative Commons Attribution 4.0 International License. Read Full License 


\section{Abstract}

\section{Background}

Much remains unknown about COVID-19 onset and rehabilitation's symptomatic features, especially the long-term health consequences of patients with COVID-19 who have been discharged from the hospital.

\section{Methods}

In this cohort study, we collected the first pandemic data of hospitalized patients in Wuhan from February 20 to March 31, 2020. All patients completed a 3-month follow-up after discharge. We carefully analyzed the detailed symptomatic characteristics of severe COVID-19 at illness onset and three months after discharge, compared it with non-severe patients, and used multiple logistic regression to determine potential symptomatic risk factors for severe COVID-19.

\section{Results}

A total of 932 hospitalized patients with COVID-19 were enrolled, including 52 severe cases and 880 nonsevere cases. Fever (60\%), cough (50.8\%), and fatigue (36.4\%) were the most common symptoms, followed by anorexia (21.8\%) and dyspnea (19.2\%). The median duration of fever was seven days, which was characterized by persistent low fever. The median duration of cough was 17 days, characterized by dry cough without sputum. Most dyspnea occurred on the fourth day after symptom onset, with a median duration of 16 days. The incidences of taste loss and olfactory disturbance were only $6.2 \%$ and $3.1 \%$, respectively. Multivariate logistic regression analysis showed that age over 65 years old (OR $6.52,95 \% \mathrm{Cl}$ 3.27-13.02, $P<0.0001)$, male sex $(3.71,1.90-7.26, P=0.0001)$, fever lasting for more than five days $(1.90$, 1.00-3.62, $P=0.0498)$, anorexia at onset $(2.61,1.26-5.40, P=0.0096)$, and modified Medical Research Council level above grade 2 when dyspnea occurred $(14.19,7.01-28.71, P<0.0001)$ were symptomatic risk factors for severe COVID-19. Three months after discharge from the hospital, $6.2 \%$ of patients still cough, $7.2 \%$ of patients still dyspnea, and $1.8 \%$ still fatigue, and $1.5 \%$ of patients had olfactory or taste disorders.

\section{Conclusions}

COVID-19 caused clusters of symptoms, with multiple systems involved. Specific symptomatic features at the onset of illness have predictive value for severe COVID-19. Persistent legacy symptoms are more frequent in severe COVID-19 patients.

\section{Introduction}

Coronavirus disease 2019 (COVID-19), caused by severe acute respiratory syndrome coronavirus 2 (SARS-CoV-2) infection, has spread unprecedentedly, causing an ongoing worldwide pandemic[1, 2]. This situation is still not optimistic, even though this illness's spread is basically under control in many countries[3]. Globally, as of 31 December 2020, there have been 81,475,053 confirmed cases of COVID-19, including 1,798,050 deaths, according to the WHO website data[4]. The outbreak and rapid spread of 
COVID-19 have caused tremendous pressure on medical resources and clinical treatment capacity worldwide[5].

Relevant studies have been published involving the clinical characteristics of patients with COVID-19 during the past few months. Although large case series have been described[6-8], most reports focused on the onset and early stages of the illness. Follow-up data regarding the illness course and the outcome after discharge from the hospital remain limited. Moreover, the spectrum of clinical manifestations of COVID-19 varies with diverse population demographics and prevalence of comorbidities. Besides, the relationship between symptomatic features and illness severity has not been fully elucidated. Therefore, it is essential to characterize symptomatic features and the progression of patients with COVID-19.

This study performed an updated analysis of detailed clinical characteristics and a 3-month follow-up in hospitalized COVID-19 patients in Wuhan, China. Our findings sought to disclose the symptomatic features of COVID-19 and help physicians make correct judgments of this disease's severity and prognosis.

\section{Methods}

\section{Study Design and Patients}

In this cohort study, we recruited hospitalized COVID-19 patients at the Optical Valley Branch of Maternal and Child Hospital of Hubei Province, Wuhan, which is a designated hospital to treat adult COVID-19 patients (aged $\geq 14$ years).

Between Feb 20 and Mar 31, 2020, this hospital admitted 1767 suspected COVID-19 patients. COVID-19 was diagnosed based on the National Health Commission of China (version 7)[9]. Only laboratoryconfirmed cases were included in this study. As shown in Figure 1, seven patients were excluded due to a lack of medical records. Fifty-eight patients were excluded due to a lack of contact information. A total of 277 patients were excluded due to negative results on nucleic acid and specific serum antibody detection. A total of 1425 cases were included in the list for the second round of medical history review and threemonth follow-up after discharge, of which 281 cases were lost to follow-up, and 212 cases were not completed. Finally, We included 932 laboratory-confirmed COVID-19 patients with complete follow-up data in our cohort for further analysis, consisting of 880 non-severe cases and 52 severe cases.

The Research Ethics Commission approved the study of the Optical Valley Branch of Maternal and Child Hospital and Shanghai Changzheng Hospital (2020SL007).

\section{Data collection}

Data were collected from electronic medical records managed by the Optical Valley Branch of Maternal and Child Hospital of Hubei Province. A total of 1760 hospitalized COVID-19 patients' medical records were copied and sent to the data processing center in Shanghai under the coordination of the Optical Valley Branch of Maternal and Child Hospital of Hubei Province. Demographic, clinical, laboratory, 
treatment, and outcome data were extracted from electronic medical records. All needed variables with necessary explanations were defined first by the research team. An experienced respiratory clinician team reviewed the copies of the medical records and abstracted the data. The abstracted data were entered into a computerized database, and double-entry was required for all variables. The data-entry team ensured that all data needed were collected. If the core data were missing, requests for clarification were immediately sent to the coordinators, who subsequently contacted the attending clinicians.

We designed a standardized electronic follow-up questionnaire form to supplement detailed symptomatic information and three-month follow-up after discharge, including onset symptoms, duration, dates, times and frequency of the symptoms, remaining symptoms after discharge, etc. The intact follow-up questionnaire form is shown in the Appendix. To ensure the validity of the collected data, patients who died, who were unable to cooperate or had unreliable narrators (i.e., elderly patients, dementia patients, and those with communication problems), were excluded from the cohort. The final date of the follow-up was Aug 02, 2020. All the authors checked the accuracy and completeness of the data.

Data cleaning, including logical checks, outlier checks, and variable engineering, was performed by experienced programmers. An experienced clinician assisted the variable engineering. The original variables were transformed if needed, including but were not restricted to converting continuous variables into categorical variables and combining multiple variables into single variables for information integration.

\section{Definitions}

Laboratory-confirmed COVID-19 was defined as a positive result on reverse transcriptase-polymerase chain reaction (RT-PCR) assay of nasal and pharyngeal swab specimens or specific serum antibodies for SARS-CoV-2. The severity of COVID-19 was evaluated following guidelines issued by the National Health Commission of China (version 7.0), as mentioned above[9]. Patients with at least one of the following conditions were defined as severe cases: 1 ) dyspnea (respiratory rate $\geq 30$ times/min); 2) finger oxygen saturation $\leq 93 \%$ in the resting state; 3 ) arterial oxygen partial pressure/oxygen absorption concentration $\leq 300 \mathrm{mmHg}$; and 4) pulmonary imaging showing that foci that progressed by $>50 \%$ in $24-48$ hours.

The onset date was defined as the earliest day when symptoms were noticed by patients themselves or physicians. Fever was defined as $37.3^{\circ} \mathrm{C}$ or higher on axillary temperature. Septic shock was defined according to the 2016 Third International Consensus Definition for Sepsis and Septic Shock[10]. Acute kidney injury was defined according to the KDIGO clinical practice guidelines[11]. The definition of acute respiratory distress syndrome referred to the Berlin diagnostic criteria[12]. Acute myocardial injury was defined as serum levels of ultrasensitive cardiac troponin I above the 99th percentile upper reference limit[13].

The Modified Medical Research Council (mMRC) Dyspnea Scale was defined as follows: level 0 for no breathlessness, level 1 for breathless when hurrying or walking up a hill, level 2 for breathless when walking slower than people of same age or requirement to stop when walking, level 3 for breathlessness 
or stopping walking after $\sim 100 \mathrm{~m}$ or a few minutes, and level 4 for breathless when dressing or not able to leave the house[14].

\section{Statistical analysis}

Categorical variables were described as frequencies and percentages. Continuous variables were described with mean, median, and interquartile range (IQR) values. Independent group t test or MannWhitney test was used to compare means for continuous variables depending on the data's distribution. The $\chi 2$ test or Fisher's exact test was used for the comparison of frequencies for categorical variables.

Univariate and multivariate logistic regression models were used to explore the symptomatic risk factors for severe COVID-19. Based on previous findings and clinical considerations, a total of 11 variables, including significant basic demographic data and symptomatic characteristics in univariate analysis, were included for multivariate analysis. If the difference between groups was not significant, the number of events was too small, odds ratios could not be calculated, or collinear with other variables, we excluded this variable from univariate analysis.

All analyses were performed using SAS JMP Pro 15.0. The tests with a $P$ value $<0.05$ were considered statistically significant.

\section{Results}

\section{Baseline characteristics of the study population}

The study population included 932 inpatients with laboratory-confirmed COVID-19, consisting of 52 severe cases and 880 non-severe cases. Table 1 presents detailed baseline characteristics, including demographics and comorbidities. The median age was 58 years (IQR 48-67). A total of 250 patients (26.8\%) were aged 14-49 years, 369 (39.6\%) were aged 50-64 years, and $313(33.6 \%)$ were aged over 65 years. A total of $557(59.8 \%)$ of the patients were women. Patients with higher median age (70, IQR 62$80)$, elderly patients $(\geq 65)(36 / 52,69.2 \%)$, and male patients $(35 / 52,67.3 \%)$ were more often found in the severe group than in the non-severe group. The proportions of smokers and drinkers among all patients were $13.3 \%$ and $7.8 \%$, respectively, with higher ratios in the severe group $(23.1 \%$ and $15.4 \%$, respectively, $P<0.05)$. The median body mass index of all the patients was $23.4 \mathrm{~kg} / \mathrm{m}^{2}($ IQR $21.4-25.4)$, which was not different between the severe group (24, IQR 21.2-25.7) and the non-severe group (23.4, IQR 21.4-25.4) $(P=0.6489)$. In the whole cohort, more than half of the patients had comorbidities $(52.5 \%)$, mainly hypertension (30.8\%), diabetes (14.4\%), ASCVD (8.4\%), chronic liver disease (4.2\%), and COPD (3.1\%). The severe group had a higher proportion of patients with at least one comorbidity and specific conditions, including ASCVD, COPD, and asthma.

\section{Detailed symptomatic features}


The most common presenting symptoms were fever (60\%) and cough (50.8\%), followed by fatigue (36.4\%), anorexia (21.8\%), and dyspnea (19.2\%). Compared with those in non-severe patients, the incidences of dyspnea (59.6\%) and anorexia (48.1\%) in severe patients were much higher $(P<0.0001$, Table 2). Notably, $11.6 \%$ of the cases were asymptomatic, including two severe cases.

\section{Fever}

As previously reported, fever is the most common symptom. Still, most of the patients had a persistent low fever $\left(37.3^{\circ} \mathrm{C}-38^{\circ} \mathrm{C}\right)$. Moderate fever $\left(38^{\circ} \mathrm{C}-39^{\circ} \mathrm{C}\right)$ was more common in severe patients (Table 2$)$. Severe COVID-19 patients had a higher proportion of fever than non-severe patients $(78.9 \%$ vs $58.9 \%, P=$ 0.0043). The median duration of fever was 7 days (IQR 4-11 days) in the non-severe group and 9 days (IQR 6-12 days) in the severe group (Figure 2, Table S1).

\section{Cough}

Most patients complained of dry cough, and white sputum was common in patients with sputum cough (Table 2). The median duration of cough was 17 days (IQR 9-35 days) for the whole cohort, while the median duration of cough in the severe patients was as long as 30 days $(12.5-51.5$ days, $P<0.05)$, almost double that of non-severe patients (Figure 2, Table S1).

\section{Dyspnea}

Dyspnea is a characteristic symptom of severe COVID-19. In our cohort, dyspnea in severe patients occurred later, lasted for a longer time, and reached higher mMRC levels (Figure 3). As shown in Table S1, the median time from illness onset to dyspnea was 4 days (IQR 1-7 days) in all patients, with 3 days (1-7 days) in non-severe patients and 7 days (1-12 days) in severe patients. The most severe dyspnea was observed on the 8th day from the beginning in non-severe patients and on the 15th day in severe patients with the disease's progression. The median duration of dyspnea in severe patients was as long as 28 days (IQR 14-51 days), which was significantly longer than that in non-severe patients (15 days, IQR 8-31 days). As shown in Table 2, severe patients showed a higher degree of dyspnea on disease onset, as quantified by mMRC levels. At the peak stage, $42.3 \%$ of the severe patients' mMRC levels reached grade 4. Even when dyspnea was relieved considerably on the 43rd day from the onset, $26.9 \%$ of the whole cohort's mMRC level was still at grade 2 .

\section{Fatigue and anorexia}

Fatigue and anorexia often coincided with fever and lasted longer than dyspnea (Figure 3). The median duration of fatigue was 14 days (8-27 days) in the non-severe group and 32 days (21-44 days) in the severe group. The median duration of anorexia was 12 days (8-24 days) in the non-severe group and 27 days (17-40 days) in the severe group ( $P<0.001$, Table $S 1) .14 .5 \%$ of the patients had anorexia at the onset, with a larger proportion in the severe group than in the non-severe group ( $30.8 \%$ vs $13.5 \%, P=$ 0.0006, Table S2). 


\section{Nausea or vomiting, and diarrhea}

Nausea or vomiting and diarrhea were not common. As shown in Table 2, only 32/932 (3.4\%) patients experienced nausea or vomiting. A total of $8.6 \%$ of patients complained of diarrhea, most of which began on the third day after illness onset, and the median duration was 5 days (IQR 4-12 days). There was no significant difference in the incidence of these symptoms between the severe and non-severe groups (Table S1).

\section{Taste loss and olfactory disturbance}

Unexpectedly, the incidence of taste loss and smell loss in our cohort was only $6.2 \%$ and $3.1 \%$, respectively (Table 2). The median duration of both symptoms was 15 days. However, the median duration of these two symptoms in severe patients (22 days and 27 days, respectively) were longer than those of non-severe patients (both were 14 days) (Figure 2, Table S1).

\section{Symptom clusters}

Cluster analysis of symptom correlation showed that specific symptoms were positively related and formed several groups (Figure 4). Anorexia, nausea or vomiting, and diarrhea formed a gastrointestinal symptom cluster. Headache and dizziness formed a neuropsychiatric symptom cluster. Loss of taste and smell were linked and often co-occurred, which formed an otorhinolaryngological symptom cluster. Fatigue and myalgia or arthralgia were highly correlated. In addition to nausea or vomiting and diarrhea, symptoms associated with anorexia included loss of taste, fatigue, and dyspnea. The only sign highly related to dyspnea was anorexia.

\section{Onset symptoms}

As shown in Table S2, fever and cough are the two most common symptoms. Only $17.3 \%$ of severe patients had dyspnea at onset, while $30.8 \%$ had anorexia. As shown in Table S3, a total of $82.7 \%$ of severe patients and $63.3 \%$ of non-severe patients started with systemic symptoms, including fever, fatigue, myalgia or joint pain. $32.7 \%$ of severe patients started with gastrointestinal symptoms, including anorexia, nausea, vomiting or diarrhea, which was significantly higher than that of non-severe patients.

\section{Symptomatic predictors of severe illness}

To explore clinical symptoms' predictive effect on the disease severity, we conducted multivariate regression analysis involving variables significantly different in the two groups (Table 3 ). The results revealed that age over 65 years old (OR 6.52, 95\% Cl 3.27-13.02, $P<0.0001)$, male sex $(3.71,1.90-7.26$, $\mathrm{P}=0.0001)$, fever lasting for more than 5 days $(1.90,1.00-3.62, P=0.0498)$, anorexia at onset $(2.61,1.26$ $5.40, P=0.0096)$, and mMRC level above grade 2 when dyspnea occurred $(14.19,7.01-28.71, P<0.0001)$ were symptomatic risk factors for severe COVID-19.

\section{Rehabilitation symptoms at 3 months after discharge}


All patients were followed up for three months after discharge (Table 4). The results showed that $6.2 \%$ of the patients still had cough, mostly dry cough. A total of $1.8 \%$ of patients had fatigue, $0.8 \%$ of patients had taste loss, and $0.7 \%$ of patients had olfactory disturbance. After discharge from hospital, a total of $7.2 \%$ patients still had dyspnea, most with mMRC level 1 . As a remaining symptom, the incidence of dyspnea was higher in the severe group than in the non-severe group $(P<0.0001)$, and severe patients had a higher degree of mMRC levels $(P<0.01)$.

\section{Discussion}

While numerous studies have reported the clinical characteristics of COVID-19 patients, this disease's symptomatic features are still not thoroughly investigated. With this study, we deeply examined the clinical data of COVID-19 patients. We uncovered more detailed information on the symptomatic features, which is essential for physicians to grasp the characteristics of COVID-19 and make a correct judgment of the illness. We found that the symptoms of COVID-19 are usually not typical but diverse. The most common presenting symptoms were fever $(60 \%)$, cough (50.8\%), and fatigue (36.4\%), which is consistent with previous reports $[15,16]$. However, the prevalence of olfactory and taste disorders (OTDs) is much lower in our cohort than in other reports[17-20]. It should be noted that $11.6 \%$ of the COVID-19 patients, including two severe cases, were asymptomatic, suggesting that the screening of COVID-19 based on symptoms is not reliable.

Although fever is the most common symptom, most patients present with only persistent low fever; of note, nearly $40 \%$ of the patients did not have a fever during the illness course. Additionally, high fever (> $39^{\circ} \mathrm{C}$ ) was rare in these patients. However, it should be mentioned that severe COVID-19 patients were more prone to suffer fever with a longer duration. Dry cough was also one of our patients' most common symptoms, which usually lasted a much more extended period than fever. As expected, the time of cough in severe patients was much longer than that in non-severe patients.

We know that dyspnea is a kind of subjective experience common in severe pneumonia patients. The occurrence of dyspnea appeared late and atypically, not always following a decline in blood oxygen saturation. Our study showed that the interval time from illness onset to dyspnea occurrence in severe patients was longer than that in non-severe patients (7 days vs. three days), presumedly caused by dyspnea's insensitivity in critical patients. In conflict with the illness severity, 40.4\% of severe COVID-19 patients did not claim dyspnea. This phenomenon is called "happy hypoxia "[21] or "silent hypoxia" [22], which means people are unaware they are being deprived of oxygen. A hypothesis was proposed that SARS-CoV-2 infection-mediated inflammation of the nucleus tractus solitarius affected the brain's hypoxia perception ability[23]. Therefore, it seems that the value of dyspnea as an alert sign for severe COVID-19 was limited.

Fatigue and anorexia also had relatively high incidence rates $(36.4 \%$ and $21.8 \%$, respectively). Onset symptoms with anorexia were associated with severe illness. This was probably because insufficient food intake in patients with anorexia resulted in malnutrition and hypoproteinemia[24], which are 
potential risk factors for severe pneumonia. The significance of anorexia varied in different age groups of COVID-19 patients. It has been reported that anorexia could be the unique onset symptom in one-third of SARS-COV-2-infected children in the UK[25].

Initially, loss of smell and taste were not considered essential symptoms for COVID-19 patients.

Nevertheless, they were defined as commonly recognized features in later reports from Western countries. In a cross-sectional survey of the prevalence of OTDs, 33.9\% (20/59) of SARS-CoV-2-positive hospitalized patients presented at least taste or olfactory disorder, and $18.6 \%$ of cases had both disorders[17]. Lechien et al. found that hospitals reported olfactory and gustatory dysfunctions, respectively[18]. However, OTDs reported in COVID-19 patients were much rarer in Asian people, ranging from 5.1-15.7\%[26, 27]. In this report, the incidence rates of dysgeusia and dysosmia were only $6.2 \%$ and $3.1 \%$, respectively, which were markedly lower than those from Western countries. There are some possible reasons for this issue. The clinical spectrum of COVID-19 is broad and varies according to ethnicity, which can be perfectly defined by the remarkable difference in the incidence rate of OTDs in different regions. It has been reported that SARS-CoV-2 has two major lineages, designated $L$ and $S$. The prevalent lineage of SARS-CoV-2 in China was different from that in Europe. The $L$ lineage was more prevalent than the $S$ lineage in the early outbreak of COVID - 19 in Wuhan, China[28]. Various SARS-CoV-2 lineages with large molecular divergence might cause distinct clinical symptoms.

It has been reported that older age, high SOFA score, and d-dimer greater than $1 \mu \mathrm{g} / \mathrm{mL}$ are risk factors for COVID-19 patients with poor prognosis at an early stage[16]. In this study, we also found that the risk factors for severe illness included age over 65 , male sex, fever lasting for more than 5 days, anorexia at the onset, and mMRC level above grade 2 . These data implied an underlying predictive value of specific clinical symptoms for the severity degree classification of COVID-19 patients. Further exploration of the symptom-based prediction model on the illness classification of COVID-19, if possible, would help health workers triage patients and recognize patients with COVID-19 requiring hospital admission. It will also be useful for doctors to screen patients inclined to develop the severe illness at an early stage and adopt intensive treatment before it is too late.

In this study, we also followed up with the patients for three months after discharge. Most non-severe and severe patients showed favorable prognoses. Cough, fatigue, and dyspnea were the most common remaining symptoms in convalescent COVID-19 patients. Some patients still did not recover from taste and olfactory disturbance. Compared with non-severe patients, severe patients were more likely to have remaining symptoms after discharge, especially dyspnea.

Our study has some limitations. As a retrospective study, some cases had incomplete documentation of the medical history and clinical manifestation. We had limited time to extract the prospective data in emergent situations. Furthermore, in our cohort, most patients (94.4\%) were non-severe cases. All confirmed COVID-19 cases were required to be admitted to designated hospitals or temporary cabin hospitals in China. Thus, the proportion of mild and moderate COVID-19 patients in Chinese hospitals was higher than that in other countries. 


\section{Conclusions}

This study reported detailed symptomatic features in severe and non-severe patients with COVID-19 in Wuhan, China. The prevalence and duration of COVID-19-related symptoms have been documented. The analysis of the relationship between clinical symptoms and illness severity revealed a cluster of predictors for COVID-19. Our study also showed an optimistic prognosis in most patients through three months of follow-up after discharge.

\section{Abbreviations}

COVID-19: Coronavirus disease 2019; MERS: Middle East respiratory syndrome; SARS: Severe acute respiratory syndrome; SARS-CoV-2: Severe acute respiratory syndrome coronavirus 2; WHO: World Health Organization; ASCVD: Atherosclerotic cardiovascular disease ; COPD: Chronic obstructive pulmonary disease

\section{Declarations}

\section{Acknowledgments}

We thank Prof. Xiao-Fei Ye, Department of Health Statistics, Second Military Medical University, for his advice regarding the clinical study, data analysis, and manuscript preparation. We thank all the patients who consented to donate their data for analysis and all the medical staff members on the front line caring for patients.

\section{Authors' contributions}

All authors had full access to all the data in the study and took responsibility for the data integrity and data analysis accuracy. WX and $\mathrm{HH}$ designed the study and revised the manuscript. LS and JW drafted the manuscript. LS and YW performed the analysis. LS, YW, ZZ, WC, BN, CY, JW, QW, CW, YL, CC, and JY collected the data. LS, $\mathrm{PH}$, and $\mathrm{YH}$ designed the electronic follow-up questionnaire form.

\section{Funding}

There was no funding source for this study. The corresponding author had the final decision to submit for publication.

\section{Availability of data and materials}

All data is available by application to the study authors.

\section{Ethics approval and consent to participate}

The study protocol was approved by the Optical Valley Branch of Maternal and Child Hospital and Shanghai Changzheng Hospital (2020SL007). Written informed consent was waived by the Ethics 
Commission of the designated hospital for emerging infectious diseases.

\section{Consent for publication}

Not applicable.

\section{Competing interests}

The authors declare that they have no conflicts of interest.

\section{References}

1. Zhu N, Zhang D, Wang W, Li X, Yang B, Song J, Zhao X, Huang B, Shi W, Lu R et al: A Novel Coronavirus from Patients with Pneumonia in China, 2019. N Engl J Med 2020, 382(8):727-733.

2. Wu F, Zhao S, Yu B, Chen YM, Wang W, Song ZG, Hu Y, Tao ZW, Tian JH, Pei YY et al: A new coronavirus associated with human respiratory disease in China. Nature 2020, 579(7798):265-269.

3. Coronavirus disease (COVID-2019) situation reports. [ https://www.who.int/emergencies/diseases/novel-coronavirus-2019/situation-reports.]

4. Coronavirus disease (COVID-19) outbreak [ https://covid19.who.int/.]

5. Ranney ML, Griffeth V, Jha AK: Critical Supply Shortages - The Need for Ventilators and Personal Protective Equipment during the Covid-19 Pandemic. N Engl J Med 2020, 382(18):e41.

6. Guan WJ, Ni ZY, Hu Y, Liang WH, Ou CQ, He JX, Liu L, Shan H, Lei CL, Hui DSC et al: Clinical Characteristics of Coronavirus Disease 2019 in China. N Engl J Med 2020, 382(18):1708-1720.

7. Wu Z, McGoogan JM: Characteristics of and Important Lessons From the Coronavirus Disease 2019 (COVID-19) Outbreak in China: Summary of a Report of 72314 Cases From the Chinese Center for Disease Control and Prevention. JAMA 2020, 323(13):1239-1242.

8. Richardson S, Hirsch JS, Narasimhan M, Crawford JM, McGinn T, Davidson KW, and the Northwell CRC, Barnaby DP, Becker LB, Chelico JD et al: Presenting Characteristics, Comorbidities, and Outcomes Among 5700 Patients Hospitalized With COVID-19 in the New York City Area. JAMA 2020.

9. New coronavirus pneumonia prevention and control program (7th edn) [http://www.nhc.gov.cn/yzygj/s7653p/202003/46c9294a7dfe4cef80dc7f5912eb1989.shtml.]

10. Rhodes A, Evans LE, Alhazzani W, Levy MM, Antonelli M, Ferrer R, Kumar A, Sevransky JE, Sprung CL, Nunnally ME et al: Surviving Sepsis Campaign: International Guidelines for Management of Sepsis and Septic Shock: 2016. Intensive care medicine 2017, 43(3):304-377.

11. Kidney disease: improving global outcomes (KDIGO) acute kidney injury work group KDIGO clinical practice guideline for acute kidney injury. [https://kdigo.org/wp-content/uploads/2016/10/KDIGO2012-AKI-Guideline-English.pdf]

12. Ranieri VM, Rubenfeld GD, Thompson BT, Ferguson ND, Caldwell E, Fan E, Camporota L, Slutsky AS: Acute respiratory distress syndrome: the Berlin Definition. Jama 2012, 307(23):2526-2533. 
13. Gao C, Wang Y, Gu X, Shen X, Zhou D, Zhou S, Huang JA, Cao B, Guo Q: Association Between Cardiac Injury and Mortality in Hospitalized Patients Infected With Avian Influenza A (H7N9) Virus. Critical care medicine 2020, 48(4):451-458.

14. Rajala K, Lehto JT, Sutinen E, Kautiainen H, Myllärniemi M, Saarto T: mMRC dyspnoea scale indicates impaired quality of life and increased pain in patients with idiopathic pulmonary fibrosis. ERJ open research 2017, 3(4).

15. Huang C, Wang Y, Li X, Ren L, Zhao J, Hu Y, Zhang L, Fan G, Xu J, Gu X et al: Clinical features of patients infected with 2019 novel coronavirus in Wuhan, China. Lancet (London, England) 2020, 395(10223):497-506.

16. Zhou F, Yu T, Du R, Fan G, Liu Y, Liu Z, Xiang J, Wang Y, Song B, Gu X et al: Clinical course and risk factors for mortality of adult inpatients with COVID-19 in Wuhan, China: a retrospective cohort study. Lancet (London, England) 2020, 395(10229):1054-1062.

17. Giacomelli A, Pezzati L, Conti F, Bernacchia D, Siano M, Oreni L, Rusconi S, Gervasoni C, Ridolfo AL, Rizzardini G et al: Self-reported Olfactory and Taste Disorders in Patients With Severe Acute Respiratory Coronavirus 2 Infection: A Cross-sectional Study. Clinical infectious diseases : an official publication of the Infectious Diseases Society of America 2020, 71(15):889-890.

18. Lechien JR, Chiesa-Estomba CM, De Siati DR, Horoi M, Le Bon SD, Rodriguez A, Dequanter D, Blecic S, El Afia F, Distinguin L et al: Olfactory and gustatory dysfunctions as a clinical presentation of mild-tomoderate forms of the coronavirus disease (COVID-19): a multicenter European study. European archives of oto-rhino-laryngology : official journal of the European Federation of Oto-RhinoLaryngological Societies (EUFOS) : affiliated with the German Society for Oto-Rhino-Laryngology Head and Neck Surgery 2020, 277(8):2251-2261.

19. Moein ST, Hashemian SM, Mansourafshar B, Khorram-Tousi A, Tabarsi P, Doty RL: Smell dysfunction: a biomarker for COVID-19. International forum of allergy \& rhinology 2020, 10(8):944950.

20. Yan CH, Faraji F, Prajapati DP, Boone CE, DeConde AS: Association of chemosensory dysfunction and COVID-19 in patients presenting with influenza-like symptoms. International forum of allergy \& rhinology 2020, 10(7):806-813.

21. Couzin-Frankel J: The mystery of the pandemic's 'happy hypoxia'. Science (New York, NY) 2020, 368(6490):455-456.

22. Chandra A, Chakraborty U, Pal J, Karmakar P: Silent hypoxia: a frequently overlooked clinical entity in patients with COVID-19. BMJ case reports 2020, 13(9).

23. U RA, Verma K: Happy Hypoxemia in COVID-19-A Neural Hypothesis. ACS chemical neuroscience 2020, 11(13):1865-1867.

24. Aziz M, Fatima R, Lee-Smith W, Assaly R: The association of low serum albumin level with severe COVID-19: a systematic review and meta-analysis. Critical care (London, England) 2020, 24(1):255.

25. Loss of appetite could be a sign of coronavirus in children as scientists find more than a THIRD of under-18s with the disease skip meals and most do not show classic symptoms. 
[https://www.dailymail.co.uk/news/article-8705465/Is-child-skipping-meals-coronavirus.html.]

26. Mao L, Jin H, Wang M, Hu Y, Chen S, He Q, Chang J, Hong C, Zhou Y, Wang D et al: Neurologic Manifestations of Hospitalized Patients With Coronavirus Disease 2019 in Wuhan, China. JAMA neurology 2020, 77(6):683-690.

27. Lee Y, Min P, Lee S, Kim SW: Prevalence and Duration of Acute Loss of Smell or Taste in COVID-19 Patients. Journal of Korean medical science 2020, 35(18):e174.

28. Tang X, Wu C, Li X, Song Y, Yao X, Wu X, Duan Y, Zhang H, Wang Y, Qian Z et al: On the origin and continuing evolution of SARS-CoV-2. National Science Review 2020, 7(6):1012-1023.

\section{Tables}


Table 1.

Baseline characteristics of 932 patients with COVID-19.

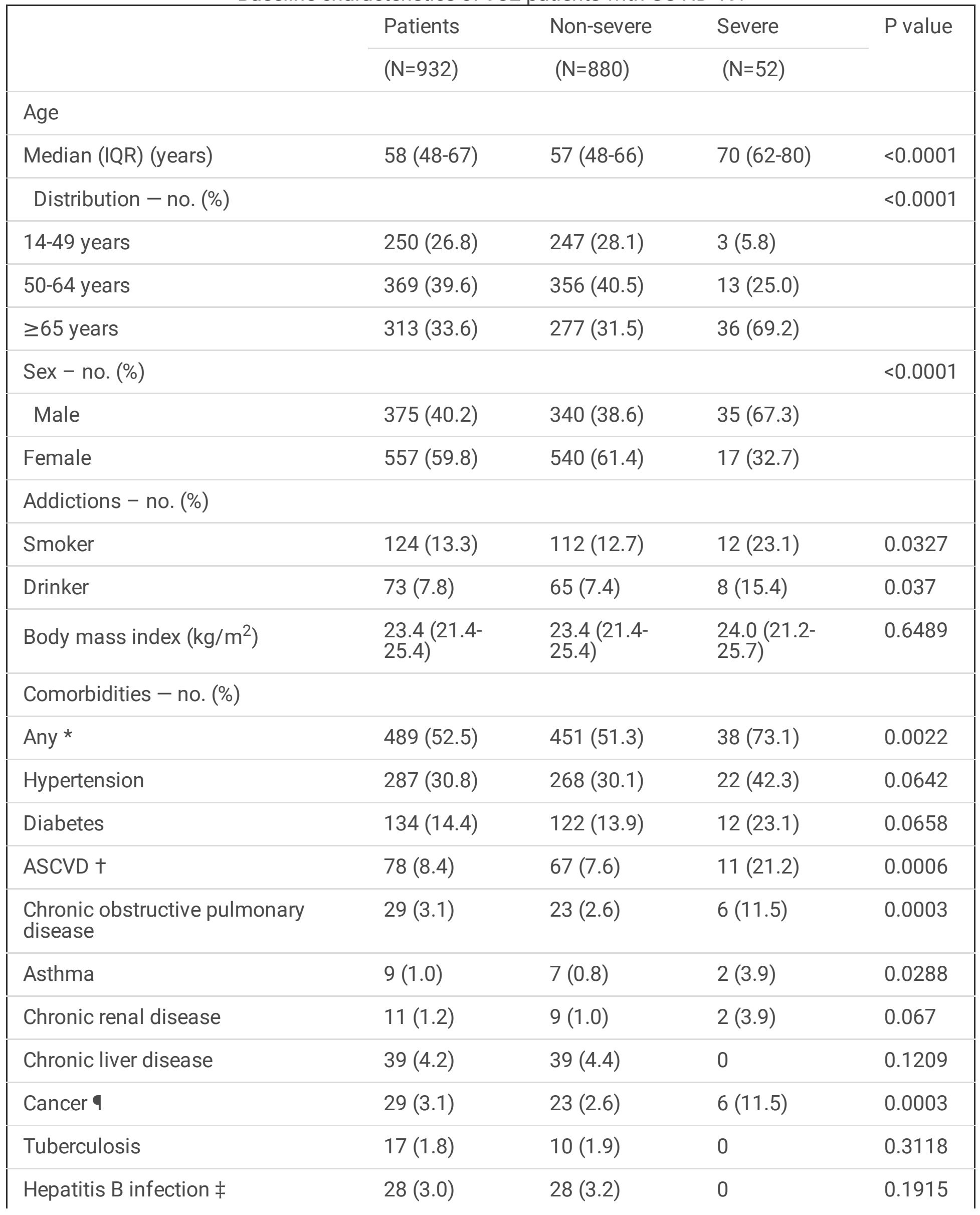


Data are median (IQR), $\mathrm{n}(\%)$, or $\mathrm{n} / \mathrm{N}(\%)$. p values were calculated by the Mann-Whitney U test, $\chi 2$ test, or Fisher's exact test, as appropriate. * $\chi 2$ test comparing all subcategories.* Included in this category is any type of comorbidity. + ASCVD = arteriosclerotic cardiovascular disease. $\neq$ The presence of hepatitis B infection was defined as a positive result on testing for hepatitis B surface antigen with or without elevated levels of alanine or aspartate aminotransferase. 9 Included in this category is any type of cancer. 
Table 2.

Symptomatic features of 932 patients with COVID-19 at admission to the hospital.

\begin{tabular}{|c|c|c|c|c|}
\hline & Patients & Non-severe & Severe & $P$ value \\
\hline & $(\mathrm{N}=932)$ & $(\mathrm{N}=880)$ & $(\mathrm{N}=52)$ & \\
\hline Fever & $559(60.0)$ & $518(58.9)$ & $41(78.9)$ & 0.0043 \\
\hline Fever type & & & & 0.3179 \\
\hline Persistent fever & $\begin{array}{l}491 / 559 \\
(87.8)\end{array}$ & $\begin{array}{l}457 / 518 \\
(88.2)\end{array}$ & $\begin{array}{l}34 / 41 \\
(82.9)\end{array}$ & \\
\hline Intermittent fever & $\begin{array}{l}68 / 559 \\
(12.2)\end{array}$ & $\begin{array}{l}61 / 518 \\
(11.8)\end{array}$ & $\begin{array}{l}7 / 41 \\
(17.1)\end{array}$ & \\
\hline Maximum body temperature & & & & 0.0002 \\
\hline $37.3-38^{\circ} \mathrm{C}$ & $\begin{array}{l}241 / 559 \\
(43.1)\end{array}$ & $\begin{array}{l}234 / 518 \\
(45.2)\end{array}$ & $\begin{array}{l}7 / 41 \\
(17.1)\end{array}$ & \\
\hline $38.1-39^{\circ} \mathrm{C}$ & $\begin{array}{l}252 / 559 \\
(45.1)\end{array}$ & $\begin{array}{l}229 / 518 \\
(44.2)\end{array}$ & $\begin{array}{l}23 / 41 \\
(56.1)\end{array}$ & \\
\hline$>39^{\circ} \mathrm{C}$ & $\begin{array}{l}66 / 559 \\
(11.8)\end{array}$ & $\begin{array}{l}55 / 518 \\
(10.6)\end{array}$ & $\begin{array}{l}11 / 41 \\
(26.8)\end{array}$ & \\
\hline Cough & $473(50.8)$ & $440(50.0)$ & $33(63.5)$ & 0.0592 \\
\hline Cough type & & & & 0.4472 \\
\hline Dry cough & $\begin{array}{l}369 / 473 \\
(78.0)\end{array}$ & $\begin{array}{l}345 / 440 \\
(78.4)\end{array}$ & $\begin{array}{l}24 / 33 \\
(72.7)\end{array}$ & \\
\hline Sputum cough & $\begin{array}{l}104 / 473 \\
(22.0)\end{array}$ & $\begin{array}{l}95 / 440 \\
(21.6)\end{array}$ & $\begin{array}{l}9 / 33 \\
(27.3)\end{array}$ & \\
\hline Sputum type & & & & 0.1253 \\
\hline White sputum & $\begin{array}{l}72 / 104 \\
(69.2)\end{array}$ & $68 / 95(71.6)$ & $4 / 9(44.4)$ & \\
\hline Yellow sputum & $\begin{array}{l}29 / 104 \\
(27.9)\end{array}$ & $25 / 95(2.8)$ & $4 / 9(44.4)$ & \\
\hline Other & $3 / 104(2.9)$ & $2 / 95(0.2)$ & $1 / 9(11.1)$ & \\
\hline Dyspnea & $179(19.2)$ & $148(16.8)$ & $31(59.6)$ & $<0.0001$ \\
\hline mMRC level at the time of dyspnea onset & & & & 0.0003 \\
\hline Level 0 & $\begin{array}{l}29 / 179 \\
(16.2)\end{array}$ & $\begin{array}{l}29 / 148 \\
(19.6)\end{array}$ & 0 & \\
\hline Level 1 & $\begin{array}{l}65 / 179 \\
(36.3)\end{array}$ & $\begin{array}{l}58 / 148 \\
(39.2)\end{array}$ & $\begin{array}{l}7 / 31 \\
(22.6)\end{array}$ & \\
\hline
\end{tabular}


Level 2

$39 / 179$

(21.8)

$28 / 148$

(18.9)

$11 / 31$

Level 3

$28 / 179$

$17 / 148$

(35.5)

Level 3

(15.6)

(11.5)

$11 / 31$

Level 4

$18 / 179$

(10.1)

$16 / 148$

(10.8)

(35.5)

mMRC level at the time of the most severe dyspnea

Level 0

$7 / 179(3.9) \quad 7 / 148(4.7) \quad 0$

Level 1

$25 / 179$

(14.0)

$25 / 148$

(16.9)

Level 2

$34 / 179$

(19.0)

$31 / 148$

(21.0)

Level 3

$54 / 179$

$(30.2)$

$48 / 148$

(32.4)

$2 / 31(6.5)$

$59 / 179$

(33.0)

$37 / 148$

(25.0)

$<0.0001$

Level 4

mMRC level at the time of dyspnea relief

Level 0

$80 / 179$

(44.7)

Level 1

$56 / 179$

(31.3)

Level 2

$34 / 179$

(19.0)

Level 3

7/179 (3.9)

$78 / 148$

(52.7)

Level 4

Fatigue

Myalgia or arthralgia

Anorexia

Loss of taste

Loss of smell

Nausea or vomiting

Diarrhea

Sore throat

Rhinorrhea

$2 / 179(1.1) \quad 0$

$47 / 148$

(31.8)

$9 / 31$

(29.0)

(19.4)

$3 / 31(9.7)$

$20 / 148$

$14 / 31$

(13.5)

(45.2)

(71.0)

2/31 (6.5)

$<0.0001$

$4 / 31$

(12.9)

2/31 (6.5)

$339(36.4) \quad 311(35.3) \quad 28(53.9) \quad 0.007$

$151(16.2) \quad 145(16.5) \quad 6(11.5) \quad 0.3476$

$203(21.8) \quad 178(20.2) \quad 25(48.1) \quad<0.0001$

$\begin{array}{llll}58(6.2) & 53(6.0) & 5(9.6) & 0.2974\end{array}$

$29(3.1)$

27 (3.1)

2 (3.9)

0.7536

$32(3.4)$

31 (3.5)

1 (1.9)

0.0809

80 (8.6)

73 (8.3)

7 (13.5)

0.2982

67 (7.2)

66 (7.5)

1 (1.9)

0.1303

$30(3.2)$

$28(3.2)$

2 (3.9)

0.792 
Headache

Dizziness

Asymptomatic infection
$38(4.1)$

33 (3.5)

108 (11.6)
$35(4.0)$

31 (3.5)

$106(12.1)$
$3(5.8)$

2 (3.9)

2 (3.9)
0.5255

0.9024

0.0727

Data are median (IQR), $\mathrm{n}(\%)$ or $\mathrm{n} / \mathrm{N}(\%)$. p values were calculated by the Mann-Whitney U test, $\chi 2$ test, or Fisher's exact test, as appropriate. ${ }^{*} \chi 2$ test comparing all subcategories. mMRC=Modified British Medical Research Council Questionnaire.

\section{Table 3.}

Symptomatic risk factors associated with severe COVID-19.

\begin{tabular}{|c|c|c|c|c|}
\hline & Univariable & $P$ value & Multivariable & $P$ value \\
\hline & \multicolumn{2}{|l|}{ OR $(95 \% \mathrm{Cl})$} & \multicolumn{2}{|l|}{ OR $(95 \% \mathrm{Cl})$} \\
\hline Male & $3.27(1.80-5.93)$ & $\begin{array}{l}<.0001 \\
0.00\end{array}$ & $3.71(1.90-7.26)$ & 0.0001 \\
\hline Age $\geq 65$ years & $\begin{array}{l}4.12(1.62- \\
10.47)\end{array}$ & 0.003 & $\begin{array}{l}6.52(3.27- \\
13.02)\end{array}$ & $\begin{array}{l}< \\
0.0001\end{array}$ \\
\hline Smoker & $2.06(1.05-4.04)$ & 0.0481 & & \\
\hline Any comorbidities & $2.58(1.38-4.83)$ & 0.0018 & & \\
\hline Dyspnea at onset & $2.66(1.25-5.72)$ & 0.0209 & & \\
\hline Anorexia at onset & $2.84(1.53-5.28)$ & 0.002 & $2.61(1.26-5.40)$ & 0.0096 \\
\hline Onset with gastrointestinal symptoms & $2.58(1.41-4.75)$ & 0.0037 & & \\
\hline Onset with systemic symptoms & $2.77(1.33-5.76)$ & 0.0027 & & \\
\hline Fever lasting for more than 5 days & $1.96(1.12-3.43)$ & 0.0191 & $1.90(1.00-3.62)$ & 0.0498 \\
\hline $\begin{array}{l}\text { mMRC grade } 2 \text { or above at dyspnea } \\
\text { onset }\end{array}$ & $\begin{array}{l}11.71(6.40- \\
21.45)\end{array}$ & <. & $\begin{array}{l}14.19(7.01- \\
28.71)\end{array}$ & $\begin{array}{l}< \\
0.0001\end{array}$ \\
\hline Dyspnea within three days of onset & $2.45(1.18-5.07)$ & 0.0258 & & \\
\hline \multicolumn{5}{|c|}{$\begin{array}{l}\text { Univariate and multivariate logistic stepwise regression models were used to explore the risk factors } \\
\text { for severe COVID-19. Based on previous findings and clinical considerations, a total of } 11 \text { variables, } \\
\text { including significant basic demographic data and symptomatic characteristics in univariate analysis, } \\
\text { were included for multivariate analysis. If the difference between groups was not significant (e.g., the } \\
\text { onset of fever) or the number of events was too small (e.g., the onset of nausea and vomiting), oddds } \\
\text { ratios could not be calculated, or they were collinear with other variables (e.g., cancer is one of the } \\
\text { comorbidities), we excluded this variable from univariate analysis. }\end{array}$} \\
\hline
\end{tabular}


Table 4.

Rehabilitation symptoms at 3 months after discharge.

\begin{tabular}{|c|c|c|c|c|}
\hline & Patients & Non-severe & Severe & $P$ value \\
\hline & $(\mathrm{N}=932)$ & $(\mathrm{N}=880)$ & $(\mathrm{N}=52)$ & \\
\hline Cough & $58(6.2)$ & $53(6.0)$ & $5(9.6)$ & 0.2974 \\
\hline Cough type & & & & 0.4501 \\
\hline Dry cough & $\begin{array}{l}43 / 58 \\
(74.1)\end{array}$ & $\begin{array}{l}40 / 53 \\
(75.5)\end{array}$ & $3 / 5$ (60.0) & \\
\hline Sputum cough & $\begin{array}{l}15 / 58 \\
(25.9)\end{array}$ & $\begin{array}{l}13 / 53 \\
(24.5)\end{array}$ & $2 / 5(40.0)$ & \\
\hline Sputum type & & & & 0.8375 \\
\hline White sputum & $\begin{array}{l}13 / 15 \\
(86.7)\end{array}$ & $\begin{array}{l}11 / 13 \\
(84.6)\end{array}$ & $2 / 2(100)$ & \\
\hline Yellow sputum & $1 / 15$ (6.7) & 1/13 (7.7) & 0 & \\
\hline Other & 1/15 (6.7) & 1/13 (7.7) & 0 & \\
\hline Fatigue & $17(1.8)$ & $15(1.7)$ & $2(3.9)$ & 0.2621 \\
\hline Loss of taste & $7(0.8)$ & $7(0.8)$ & 0 & 0.5186 \\
\hline Loss of smell & $5(0.7)$ & $5(0.7)$ & 0 & 0.5454 \\
\hline Anorexia & $13(1.4)$ & $11(1.3)$ & $2(3.9)$ & 0.1209 \\
\hline Dyspnea & $67(7.2)$ & $50(5.7)$ & $17(32.7)$ & $<0.0001$ \\
\hline $\begin{array}{l}\text { mMRC level of dyspnea after } 3 \text { months of } \\
\text { discharge }\end{array}$ & & & & 0.0067 \\
\hline Level 0 & $\begin{array}{l}24 / 67 \\
(35.8)\end{array}$ & $20 / 50(40)$ & $\begin{array}{l}4 / 17 \\
(23.5)\end{array}$ & \\
\hline Level 1 & $\begin{array}{l}30 / 67 \\
(44.8)\end{array}$ & $25 / 50(50)$ & $\begin{array}{l}5 / 17 \\
(29.4)\end{array}$ & \\
\hline Level 2 & $\begin{array}{l}10 / 67 \\
(14.9)\end{array}$ & $5 / 50(10)$ & $\begin{array}{l}5 / 17 \\
(29.4)\end{array}$ & \\
\hline Level 3 & $1 / 67(1.5)$ & 0 & $1 / 17(5.9)$ & \\
\hline Level 4 & 2/67 (3.0) & 0 & $\begin{array}{l}2 / 17 \\
(11.7)\end{array}$ & \\
\hline $\begin{array}{l}\text { Data are median (IQR) or } n(\%) \text {. p values we } \\
\text { Fisher's exact test, as appropriate. mMRC= } \\
\text { * } \chi 2 \text { test comparing all subcategories. }\end{array}$ & $\begin{array}{l}\text { eulated by th } \\
\text { ed British Me }\end{array}$ & $\begin{array}{l}\text { Mann-Whitney } \\
\text { cal Research }\end{array}$ & $\begin{array}{l}J \text { test, } \chi 2 \text { tes } \\
\text { Juncil Quest }\end{array}$ & $\begin{array}{l}\text { or } \\
\text { nnaire. }\end{array}$ \\
\hline
\end{tabular}


1767 suspected COVID-19 patients were admitted to the Optical Valley Branch of Maternal and Child Hospital of Hubei Province, Wuhan from Feb 20 to Mar 31, 2020

7 patients were lacking medical records 58 patients were lack of contact information

277 patients were excluded because of SARS-Cov-2 RNA and antibodies negative

1425 patients were included in the follow-up list

$$
\mid
$$

281 patients were lost to follow-up 171 refused to cooperate with follow-up 66 contact information error 38 cannot provide clear medical history 6 died after discharge

212 patients did not complete the follow-up

932 patients were included in the final analysis

\section{Figure 1}

The flowchart showing the strategy of participant enrollment. 


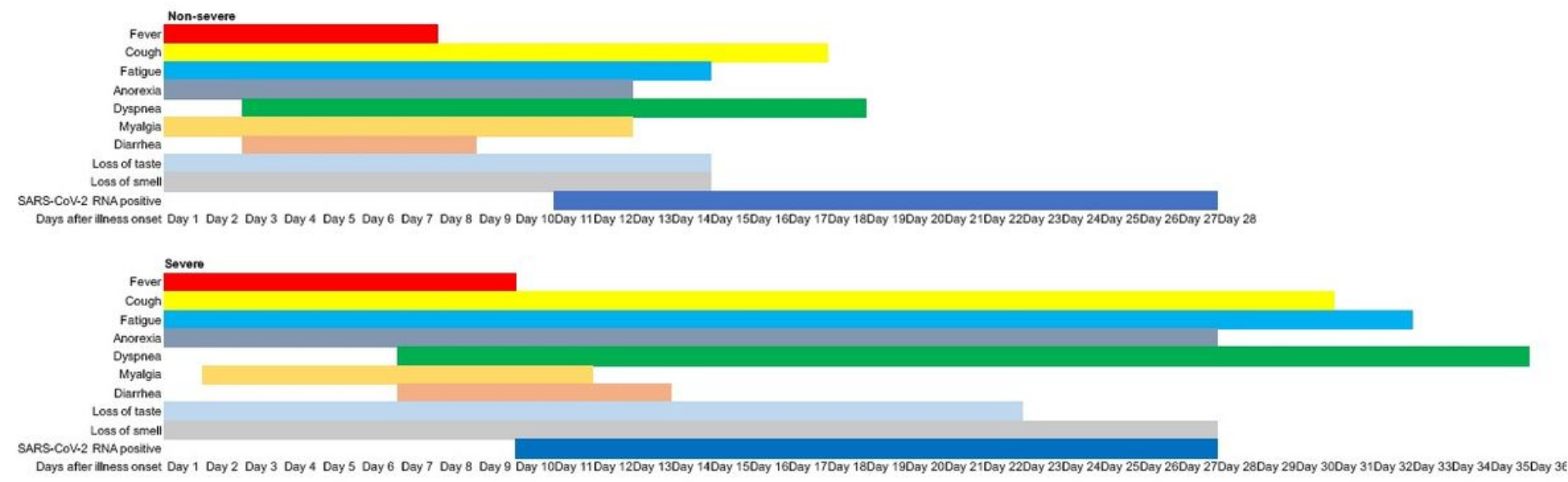

\section{Figure 2}

Clinical courses of major symptoms and duration of viral shedding from illness onset in patients hospitalized with COVID-19.

Severe : median (IQR) duration of dyspnea 28 (15-43) days ( $n=31)$

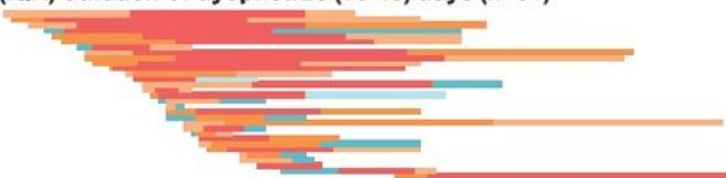

Non-severe: median (IQR) duration of dyspnea 16 (14-28) days ( $n=148)$

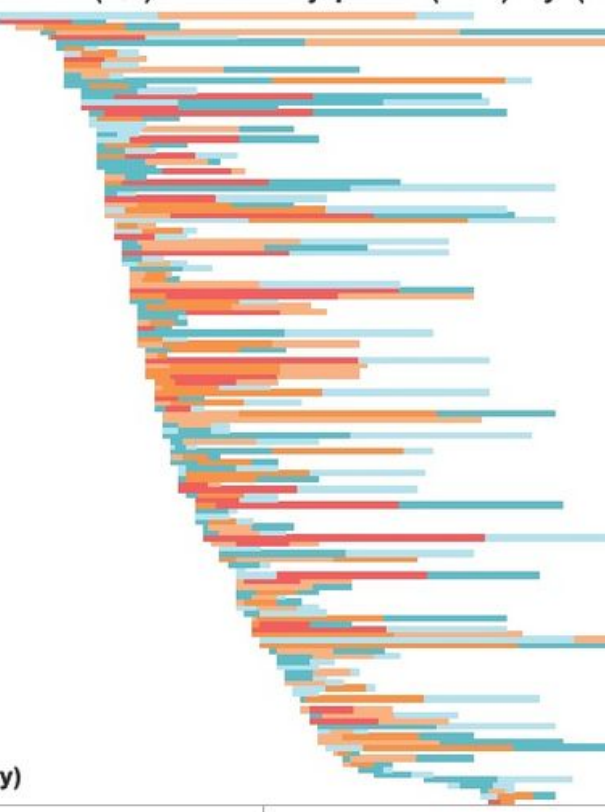

60

100

150

200

\section{Figure 3}

Timeline of patients hospitalized with COVID-19 who were dyspnea. 


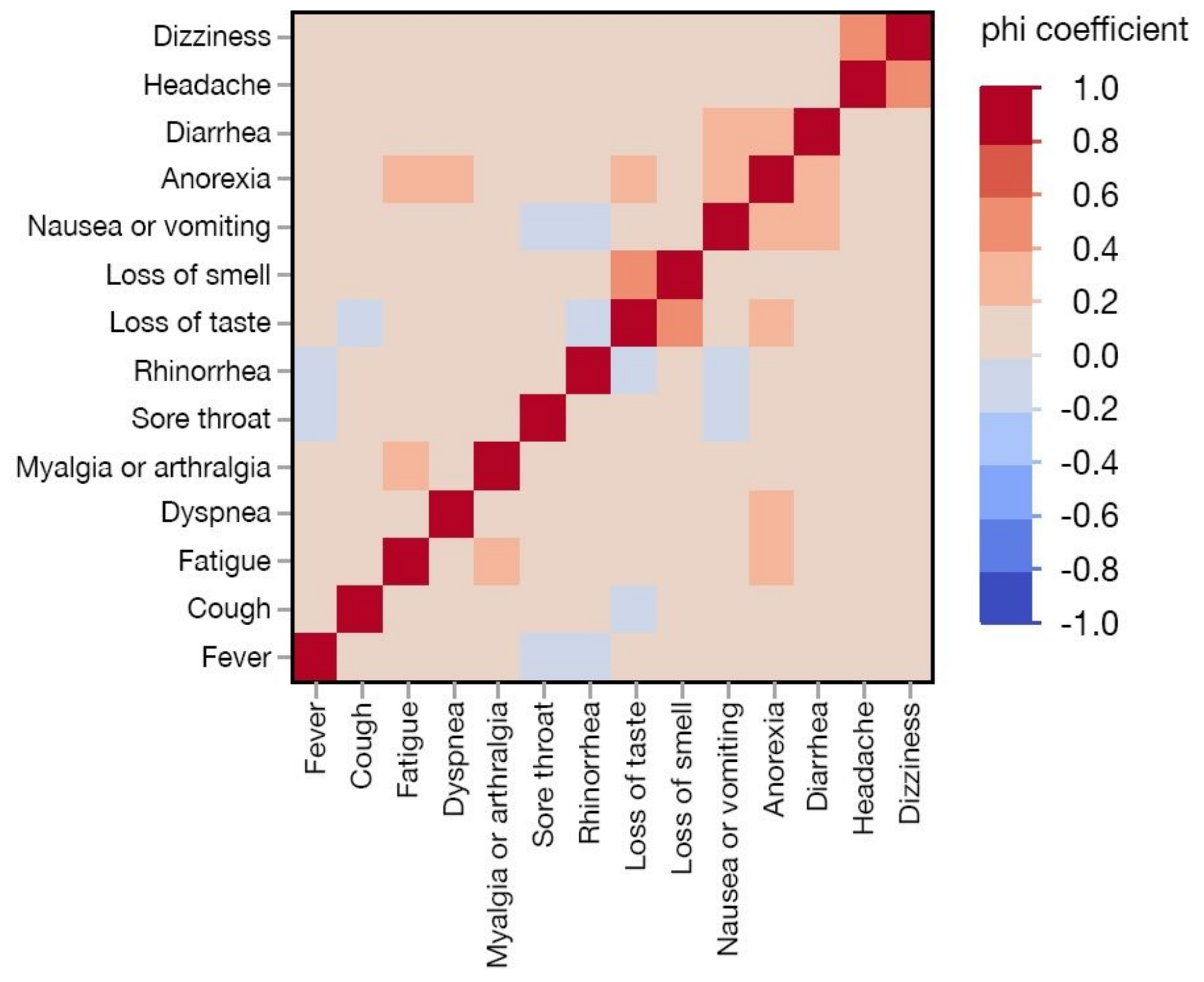

Figure 4

Heatmap for correlation between different symptoms.

\section{Supplementary Files}

This is a list of supplementary files associated with this preprint. Click to download.

- Appendix1.Studyprotocol.docx

- Appendix2.COVID19followupQuestionnaire.docx

- Appendix3.STROBEchecklistv4combined.docx

- Appendix4.Tables13.docx 